\title{
Evaluation of fatigue-ratcheting damage of a pressurised elbow undergoing damage seismic inputs
}

\author{
K. Dang Van ${ }^{\mathrm{a}, *}$, Z. Moumni ${ }^{\mathrm{b}}$ \\ ${ }^{\mathrm{a}}$ LMS Ecole Polytechnique, CNRS,UMR 7649, 91128 Palaiseau Cedex, France ${ }^{\mathrm{b}}$ \\ Laboratoire de Mécanique, ENSTA, 91760 Palaiseau Cedex, France
}

\begin{abstract}
We present a simplified method to calculate the plastic ratchet of elbow-shaped pipes submitted to seismic loading and an internal pressure. This method is simplified in the sense that the value of the ratchet is obtained without the use of finite element method (FEM) calculations. Here we derive a formula and use it to evaluate the fatigue-ratcheting damage of an elbow. This approach is applicable to complex plastic response appropriately described by non-linear kinematics hardening, which is more realistic for stainless steel such as 316-L
\end{abstract}

\section{Introduction}

Industrial structures, designed for transportation of pressurised fluids often requires high safety levels against accidental seismic loading. For example, in the case of nuclear structures, which must fulfil a criterion against fracturing, the most vulnerable components are the curved pipes because they are more flexible than straight pipes. They suffer much more severe stresses under the same bending moment. It is well known that this is due to the ovaling of the curved pipe cross section during bending. To predict their fatigue life, under seismic loading, it is necessary to calculate the local mechanical parameters that can

\footnotetext{
* Corresponding author. Tel.: + 33-1-69-33-33-73; fax: + $33-1-69-33-30-26$.
}

initiate the general collapse of a structure by low-cycle plastic fatigue.

It is very difficult to perform direct experimentation and to interpret the obtained results for many reasons.

First, it is not easy to predict the locus of crack initiation. The strain gages are rapidly de-bonded, and it becomes impossible to measure local strains. To avoid these difficulties numerical simulations are performed to evaluate the strain and stress histories within the vicinity of points of stress concentrations. These calculations present a number of difficulties since we are dealing with a shell problem presenting severe geometrical (large displacement) and material (plasticity) nonlinearities.

Boussa et al. (1996) presented an original treatment of the finite bending of curved pipes of 
arbitrary cross sections. Their model has been used to treat the previous problem (Boussa, 1992 and Boussa et al. (1994)). Nevertheless, only two simple constitutive laws have been used: perfect plasticity, and linear kinematics hardening law. The first one underestimates the ratcheting rate threshold, whereas the second is inadequate to describe this phenomenon. In addition, the calculations are rather CPU intensive, limiting our ability to perform the large number of simulations necessary to derive a design rule for a code of practice.

In this paper, we propose a simplified model that relates the strain histories at some critical points to structure characteristics and seismic loading sequences. Our method is applicable to more realistic material behaviour taking into the account non-linear kinematics hardening. It is known that this model is more realistic for $316-\mathrm{L}$ stainless steel.

The paper is organised as follows. In the second section, we give an example of calculation of the finite pure bending of a curved pipes based on Boussa et al. (1996) model. In this formulation, it is shown that the problem can be reduced to an axisymmetric problem augmented with one degree of freedom. The third section is devoted to the simplified method. First we derive a formula that gives the ratchet deformation in the case of perfect plasticity. It is then generalised for non-linear kinematic hardening laws. In the fourth section, we present the fatigue analysis based on the modified Masson-Coffin equation (Coffin, 1970) to take account of ratcheting.

\section{Numerical finite elements calculation}

We give in this sequel an example of calculation of the finite pure bending of curved pipes using Boussa-Dang Van model.

\subsection{Notation}

Throughout the paper, the symbols $\otimes$ and $\left({ }^{\circ}\right)$ denotes the standard tensor products of two vectors or two second-order tensors and the differentiation with respect to time.

\subsection{Boussa-Dang Van model}

Boussa et al. (1996), proposed a new and general formulation for solving by finite elements method (FEM) the finite pure bending of a curved pipes. In the following, the main hypotheses of their model are first recalled. Using the virtual work principle, the authors' show that the problem, after discretisation by the FEM, is reduced to an axisymmetric problem augmented by a single degree of freedom.

\subsubsection{Kinematics}

In its initial configuration assumed to be stress free, the curved pipe of interest, shown in Fig. 1 with the main notations, is supposed to be a three-dimensional body occupying a sector $\Omega_{0}$ of an axis symmetric body. Let $\Sigma_{0}$ denote a typical cross section, $\Sigma_{0}^{\text {sol }}$ being the structure of the pipe itself and $\Sigma_{0}^{\text {int }}$ being the cavity formed by this structure. In addition, we have $S_{0}^{\text {int }}$ and $S_{0}^{\text {ext }}$ presenting the inner and outer skins, $\Psi_{0}$ the bend angle of the curved pipe and $\mathscr{R}_{0}$ the radius of curvature defined by:
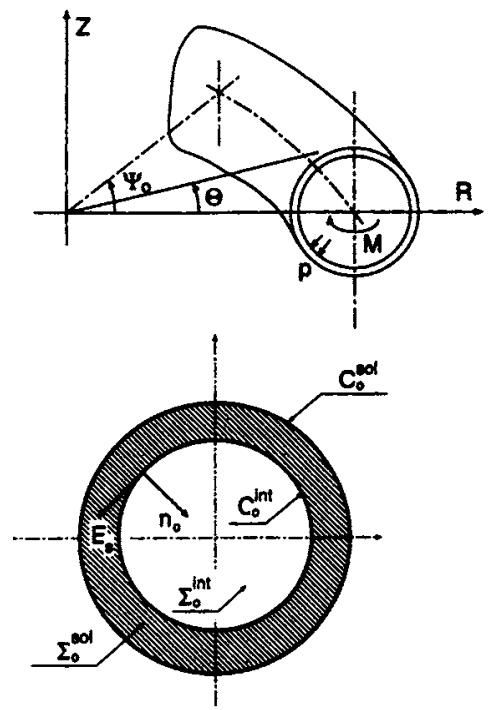

Fig. 1. General view and typical cross section of the curved pipe. 
$\mathscr{R}_{0}=\frac{\left(\int_{\Sigma_{0}} r \mathrm{~d} \Sigma_{0}\right)}{\left(\int_{\Sigma_{0}} \mathrm{~d} \Sigma_{0}\right)}$

Let $M$ be the position of a typical particle with cylindrical co-ordinates $(R, \Theta, Z)$ and a normalised natural basis $\left(E_{R}, E_{\Theta}, E_{Z}\right)$ :

$M=O+R E_{R}(\Theta)+Z E_{Z}$

Under external loads, the body deforms and a particle with initial position $M$ occupies the new position $\mathrm{m}(r, \theta, z)$ with a normalised natural basis $\left(e_{r}, e_{\theta}, e_{z}\right)$ :

$m=O+r e_{r}(\theta)+z e_{z}$

The pure in plane bending of the curved pipe can then be conveniently described with the following relations:

$$
\left\{\begin{array}{c}
r=r(R, Z, t) \\
\theta=(1+a(t)) \Theta \\
z=z(R, Z, t)
\end{array}\right.
$$

where $a(t)$ is a scalar function of time. The kinematics given by Eq. (4) includes the usual assumptions for the in-plane bending problem. The assumption that cross sections remain plane is accounted for by the dependence of $\theta$ on $\Theta$ solely, and the assumption that all the cross sections deform likewise in their plane is enforced by the independence of $r$ and $z$ from $\Theta$. Also, the assumption of uniform stretching of parallels to the axis of symmetry is the consequence of the linearity between $\theta$ and $\Theta$. In addition, fixing $a(t)$ to 0 in Eq. (4) yields an axisymmetric evolution. Accordingly, arbitrary values of $a(t)$ adds only one degree of freedom to the axisymmetric problem.

\subsubsection{External loading and boundary conditions}

In the sequel the Cauchy stress tensor will be denoted by $\underline{\sigma}$. In its initial state, the pipe is supposed stress free. The applied loads are of two types: those (typical in three-dimensional continuum descriptions) given pointwise, and those (less common) prescribed on average. In the current problem, these are the bending moment $\mathscr{M}$ due to the seismic input and the normal force $\mathscr{N}$ due to the effect of the pipe plug. They are defined on the current configuration by:

$$
\left\{\begin{aligned}
\mathscr{M}=\left[\int_{\Sigma f^{\mathrm{ol}}} G M \wedge \underline{\underline{\sigma}} e_{\theta}\right] e_{z} & =\int_{\Sigma f^{\mathrm{ol}}}\left(r-\mathscr{R}_{t}\right) \sigma_{\theta \theta} \mathrm{d} \Sigma_{t} \\
\mathscr{N}=p A^{\mathrm{int}} & =\int_{\Sigma f^{\mathrm{ol}}} \sigma_{\theta \theta} \mathrm{d} \Sigma_{t}
\end{aligned}\right.
$$

In Eq. (5) $p$ represents the internal pressure and $A^{\text {int }}$ the area of $\Sigma^{\text {int }}$. The boundary conditions are the following:

- The outer skin $S^{\text {ext }}$ is stress free:

$$
T^{\mathrm{d}}=\underline{\underline{\sigma}} n=0
$$

- The inner skin $S^{\text {int }}$ is submitted to uniform pressure:

$$
T^{\mathrm{d}}=\underline{\underline{\sigma}} n=-p n
$$

- At the end cross sections $\sum_{0}^{\mathrm{sol}}$ and $\sum_{\Theta}^{\mathrm{sol}}$ the tangential component of the applied shear force vanish:

$$
T_{r}^{\mathrm{d}}=T_{z}^{\mathrm{d}}=0
$$

Finally the end cross section $\Sigma_{\Theta}^{\text {sol }}$ is submitted to a prescribed bending moment $\mathscr{M}$ and to a normal force $\mathscr{N}$. They are given by Eq. (5). In the case of a curvature controlled loading, the moment is an unknown variable that needs to be calculated and the rotation prescribed by the condition:

$\theta=(1+a(t)) \Theta$

\subsubsection{Virtual work equation}

In the case of quasi-static evolution, the principle of Virtual Work is:

$\mathscr{P}_{\mathrm{i}}+\mathscr{P}_{\mathrm{e}}=0$

where $\mathscr{P}_{\mathrm{i}}$ and $\mathscr{P}_{\mathrm{e}}$ are respectively the power of internal and external forces. Denoting the rate of deformation by $\hat{d}$ and the virtual velocity by $\hat{\boldsymbol{v}}$ leads to: 
$-\int_{\Omega} \underline{\underline{\sigma}}: \hat{d} \mathrm{~d} \Omega+\int_{S^{\text {int }}} \hat{\boldsymbol{v}} \underline{\underline{\sigma}} n \mathrm{~d} S+\int_{\Sigma \S^{1}} \underline{\underline{v}} \underline{\underline{\sigma}} e_{\theta} \mathrm{d} \Sigma$

$-\int_{\Sigma \text { gol }} \hat{v} \underline{\underline{\sigma}} e_{\theta} \mathrm{d} \Sigma$

where $\Omega$ is the region of space occupied by the curved pipe and

$\hat{\boldsymbol{v}}=\hat{r} e_{r}+r \Theta \hat{a} e_{\theta}+\hat{z} e_{z}$

Taking into account the boundary conditions Eqs. (5)-(7) and Eq. (8), yields the following equation:

$-\int_{\Omega} \underline{\underline{\sigma}}: \hat{d} \mathrm{~d} \Omega-\int_{S^{\text {int }}} p \hat{\boldsymbol{v}} n \mathrm{~d} S+\left(\mathscr{M}+p \mathscr{R} A^{\mathrm{int}}\right) \Theta \hat{a}=0$

The constitutive law considered is the perfect plasticity given by the following equation:

$\underline{\underline{\sigma}}=\mathscr{C}:\left(d-d^{\mathrm{p}}\right)$

where $\mathscr{C}$ is the standard constant elastic modulus tensor of the material, which is assumed to be homogeneous and isotropic, and $\mathrm{d}^{p}$ the plastic deformation rate.

The total rate of deformation can be decomposed in two terms $d=d_{\Sigma}+\dot{a} e_{\theta} \otimes e_{\theta}$. The first one, $d_{\Sigma}$, corresponds to the axisymmetric evolution and the second, $\dot{a} e_{\theta} \otimes e_{\theta}$ corresponds to the deformation of the parallels.

Differentiating Eq. (12) with respect to time and taking into account, the constitutive law (Eq. (13)) and the decomposition of the total deformation the rate, yields the following equation:

$$
\begin{aligned}
& -\int_{\Omega} d_{\Sigma}: \mathscr{C}: \hat{d}_{\Sigma} \mathrm{d} \Omega-\dot{p} \int_{S_{\text {int }}} n \hat{\boldsymbol{v}}_{\Sigma}+\int_{\Omega} d^{\mathrm{p}}: \mathscr{C}: \hat{d}_{\Sigma} \mathrm{d} \Omega \\
& -\hat{a} \int_{\Omega} d_{\Sigma}: \mathscr{C}:\left(e_{\theta} \otimes e_{\theta}\right) \mathrm{d} \Omega-\dot{a} \int_{\Omega} \hat{d}_{\Sigma}: \mathscr{C}:\left(e_{\theta} \otimes e_{\theta}\right) \mathrm{d} \Omega \\
& -\dot{a} \hat{a} \int_{\Omega}\left(e_{\theta} \otimes e_{\theta}\right): \mathscr{C}:\left(e_{\theta} \otimes e_{\theta}\right) \mathrm{d} \Omega \\
& +\left(\dot{\mathscr{M}}+\dot{p} \mathscr{R} A^{\mathrm{int}}\right) \Theta \hat{a}+\hat{a} \int_{\Omega} d^{P}: \mathscr{C}:\left(e_{\theta} \otimes e_{\theta}\right) \mathrm{d} \Omega=0
\end{aligned}
$$

Further information concerning the model outlined above and its finite element discretisation is found in Boussa et al. (1996).
Table 1

Geometry and material parameters

Radius of curvature $\mathscr{R}(\mathrm{mm})$

228.6

External radius $r_{\mathrm{e}}(\mathrm{mm})$

84.15

Thickness $t$ (mm)

Young modulus $E(\mathrm{GPa})$

Elastic limit $S_{\mathrm{y}}(\mathrm{MPa})$

200

Poisson ration $v$

300

Internal pressure $p(\mathrm{MPa})$

0.28

11.7

Amplitude of the rotation $a$

\subsection{An example of calculation}

As an example, the bending of a pressurised curved pipe with perfectly plastic behaviour is presented. The computations were performed with Castem 2000 code. The geometry, material parameters and loading amplitudes are given in Table 1.

Because of symmetry, the mesh was defined only for half of the section as presented on Fig. 2. The imposed rotation history is given in Fig. 3. The outcome of the finite elements calculation is the evolution of the moment versus the rotation, given in Fig. 4. One can see that the stabilised state is reached after a few cycles only. This is due to the imposed displacement condition. Fig. 5 presents the history of the hoop strain at the critical point of the structure (internal flank) versus the imposed rotation. We observe that plastic deformation increases with the number of loading cycles. This ratcheting effect is due to the internal pressure that prevents ovalisation of the section, so that the rate of deformation is not the same during the opening and the closure sequences of the bending.

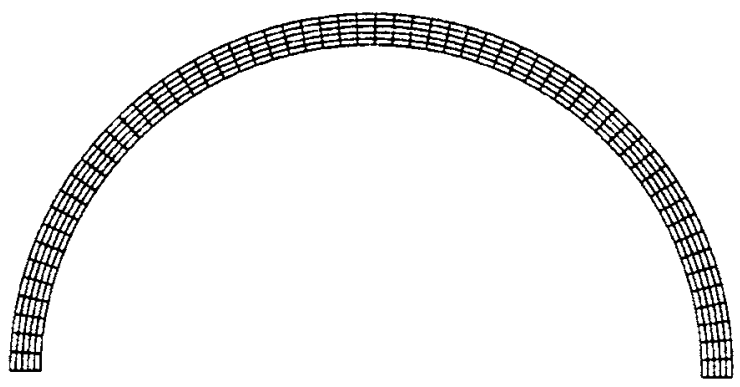

Fig. 2. Mesh over the half of the section. 


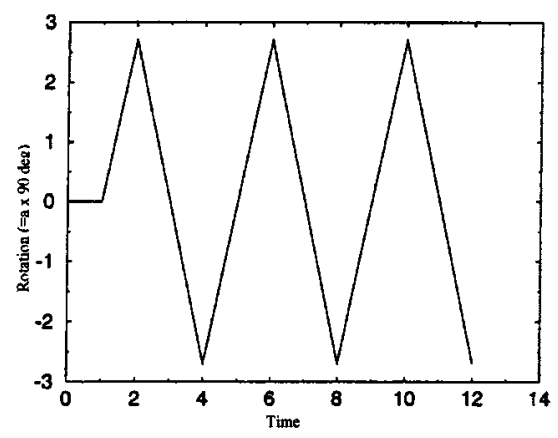

Fig. 3. Imposed rotation versus time.

The FEM computation is time consuming, so that a simplified method, which permits a greater number of simulations for a sensitivity analysis, is necessary. Such simplified model is discussed in the next section.

\section{Simplified method}

The simplified method is first presented for the same problem considered above with an elastic perfectly plastic model. It is then generalised for non-linear kinematic hardening laws.

\subsection{Evaluation of the hoop strain in the inner skin}

The simplified method is base on the analysis of the (Fig. 5). We approximate the hoop strain in the inner skin (critical point of the structure) by the bilinear curve given in the (Fig. 6).

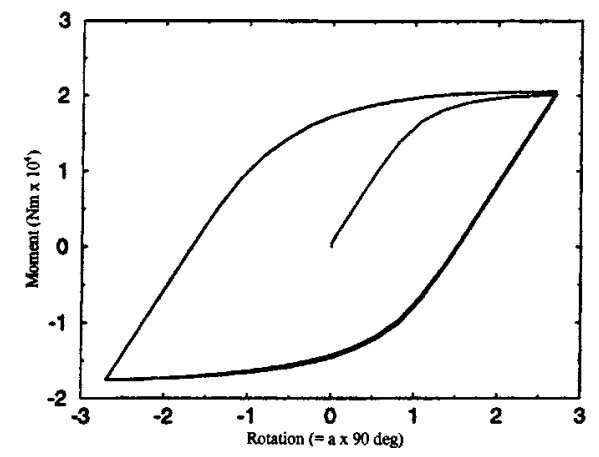

Fig. 4. Moment $\left(\mathrm{Nm} \times 10^{4}\right)$ versus imposed rotation.

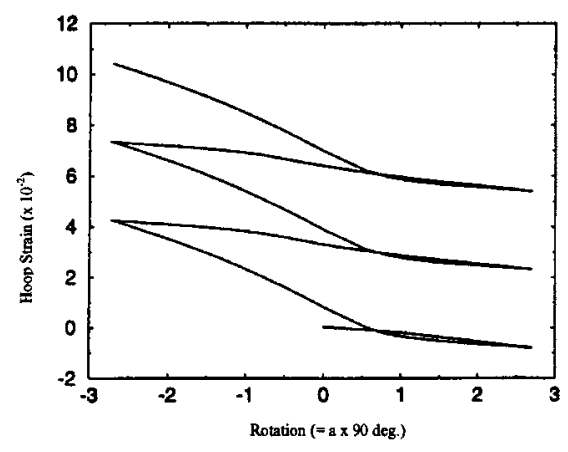

Fig. 5. Hoop strain (\%) versus imposed rotation.

The elastic range is given by the interval of rotation $\left[2 A_{\mathrm{y}}^{\mathrm{o}}, 2 A_{\mathrm{y}}^{\mathrm{c}}\right]$ where $A_{\mathrm{y}}^{\mathrm{o}}$ and $A_{\mathrm{y}}^{\mathrm{c}}$ are the elastic limits of the elbow during opening and closing, respectively. The difference between $A_{\mathrm{y}}^{\mathrm{o}}$ and $A_{\mathrm{y}}^{\mathrm{c}}$ is denoted $l$. The hoop strain varies linearly with the imposed rotation angle $A=90 \times$ $a(t)$. The slope of the curve is denoted by $\alpha$. The plastic range is characterised by two different curves with different slopes: $\beta$ for opening and $\gamma$ for closing.

Fig. 6 shows that our model is based on four parameters $\alpha, \beta, \gamma$ and $l$ that can be determined by a parametric method in the following way.

\subsubsection{Parameter $l$}

$l$ corresponds roughly to the range of initial elasticity of the inner flank:

$l=A_{\mathrm{y}}^{\mathrm{o}}-A_{\mathrm{y}}^{\mathrm{c}}$

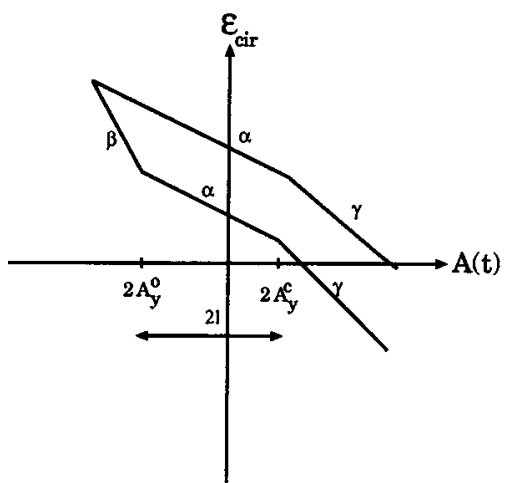

Fig. 6. Parameters of the simplified model. 
Let $a_{\mathrm{y}}$ and $\mathscr{M}_{\mathrm{y}}$ is, respectively, the limit rotation and moment that lead to the first plastification. Leconte (1992) estimates the limit moment by the formula:

$\mathscr{M}_{\mathrm{y}}=\frac{S_{\mathrm{y}} I}{r_{\mathrm{e}} C}$

where, $S_{\mathrm{y}}$ the elastic limit of the material, $I$ the moment of inertia of the section of the pipe, $r_{\mathrm{e}}$ the external radius and $C$ a stress concentration coefficient. This last coefficient is given by Clarck and Reissner (1951) as:

$C=\frac{1.8}{h^{2 / 3}}, \quad h=\frac{t \mathscr{R}}{r_{\mathrm{m}}^{2}}$

Where $r_{\mathrm{m}}$ the mean radius, and $t$ the thickness of the elbow.

Considering a linear relation between $a_{\mathrm{y}}$ and $\mathscr{M}_{\mathrm{y}}$ yields the following equation:

$a_{\mathrm{y}}=k \frac{h^{2 / 3} \mathscr{R}}{1.8 r_{\mathrm{e}} E} S_{\mathrm{y}}$

$k$ is a flexibility coefficient given by Clarck and Reissner (1951) as:

$k=\frac{1.73 \sqrt{1-v^{2}}}{h}$

Eq. (18) and Eq. (19) lead to:

$A_{\mathrm{y}}^{\mathrm{o}}=-A_{\mathrm{y}}^{\mathrm{c}}=90 \times a_{\mathrm{y}}=86.5 \frac{\mathscr{R} \sqrt{1-v^{2}}}{h^{2 / 3} r_{\mathrm{e}} E} S_{\mathrm{y}}$

The internal pressure induces initial stresses having the tendency to decrease the value of the limit rotation. These stresses are given by:

$\sigma_{\theta \theta}=\frac{p r_{\mathrm{m}}}{t}$

Considering the difference between the behaviour of the curved pipe in opening and closing (because of the internal pressure) a parametric calculation gives:

$$
\begin{aligned}
& A_{\mathrm{y}}^{\mathrm{o}}=112.4 \frac{\mathscr{R} \sqrt{1-v^{2}}}{h^{2 / 3} r_{\mathrm{e}} E}\left(S_{\mathrm{y}}-0.8 \frac{p r_{\mathrm{m}}}{t}\right) \\
& A_{\mathrm{y}}^{\mathrm{c}}=-86.5 \frac{\mathscr{R} \sqrt{1-v^{2}}}{h^{2 / 3} r_{\mathrm{e}} E}\left(S_{\mathrm{y}}-0.8 \frac{p r_{\mathrm{m}}}{t}\right)
\end{aligned}
$$

Finally $l$ is given by:

$$
l=199 \frac{\mathscr{R} \sqrt{1-v^{2}}}{h^{2 / 3} r_{\mathrm{e}} E}\left(S_{\mathrm{y}}-0.8 \frac{p r_{\mathrm{m}}}{t}\right)
$$

The three left parameters $\alpha, \beta, \gamma$ are determined following the same procedure using a parametric method based on some finite elements calculation.

\subsubsection{Parameter $\alpha$}

This parameter presents the slope of the elastic part of the curve. It depends only on the geometry of the pipe. The parametric calculation gives for $\alpha$ :

$\alpha=-\frac{1}{98.3}\left(\frac{t r_{\mathrm{m}}}{\mathscr{R}^{2}}\right)^{1 / 3}$

For the curved pipe at hand one finds: $\alpha=$ -0.00226 .

\subsubsection{Parameters $\beta, \gamma$}

They correspond to the slopes of the plastic parts of the curve for closing $(\beta)$ and opening $(\gamma)$. They depend on the geometry and on the elastic limit $S_{\mathrm{y}}$ and the pressure $p$. Their evaluation is more difficult than $\alpha$ because it is necessary to calculate the deformation response of the structure for some cycles.

It is clear that in the absence of pressure, the behaviour of the curved pipe is identical in opening and closing. The parametric calculation gives in this case:

$\beta=\gamma=3.5 \alpha$

Taking into account the internal pressure leads to different values for $\beta$ and $\gamma$. To evaluate them, we fixed the geometry and the elastic limit $S_{\mathrm{y}}$ and we calculated their variations with respect of $p$. The results are represented in (Fig. 7):

The curve given in the figure (Fig. 7) can be approximated by the following formulas:

if $\frac{p r_{\mathrm{m}}}{S_{\mathrm{y}} t}<\frac{1}{3}\left\{\begin{array}{l}\beta=\left(3.5+10.5 \frac{p r_{\mathrm{m}}}{S_{\mathrm{y}} t}\right) \alpha \\ \gamma=3.5 \alpha\end{array}\right.$ 


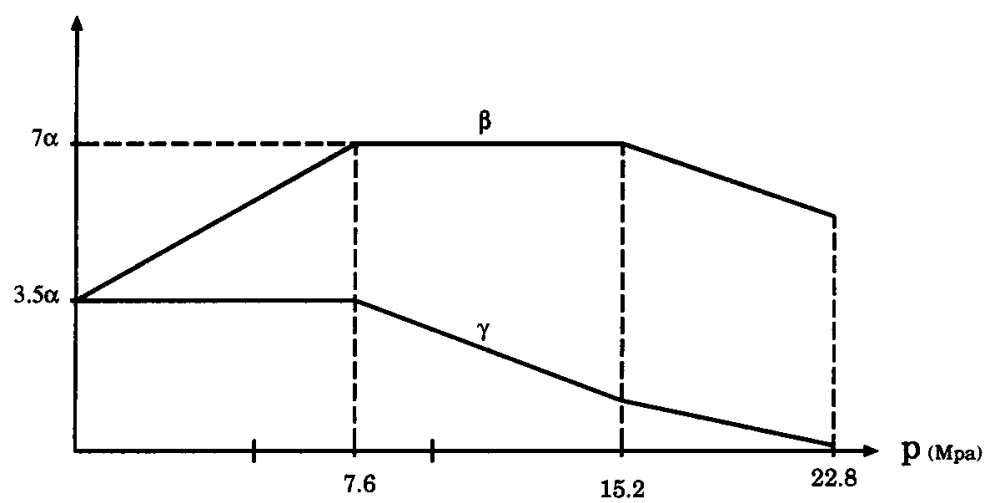

Fig. 7. Evolution of $\beta$ and $\gamma$ with respect of the internal pressure.

if $\frac{1}{3}<\frac{p r_{\mathrm{m}}}{S_{\mathrm{y}} t}<\frac{2}{3} \quad\left\{\begin{array}{l}\beta=7 \alpha \\ y=\left(6-7.5 \frac{p r_{\mathrm{m}}}{S_{\mathrm{y}} t}\right) \alpha\end{array}\right.$

if $\frac{2}{3}<\frac{p r_{\mathrm{m}}}{S_{\mathrm{y}} t}<1 \quad\left\{\begin{array}{l}\beta=\left(11-6 \frac{p r_{\mathrm{m}}}{S_{\mathrm{y}} t}\right) \alpha \\ \gamma=\left(3-3 \frac{p r_{\mathrm{m}}}{S_{\mathrm{y}} t}\right) \alpha\end{array}\right.$

In (Eq. (26)), the adimensional number $\left(p r_{\mathrm{m}}\right) /\left(S_{\mathrm{y}} t\right)$ measures the internal pressure effect.

Our method permits the calculation of the response of the structure (hoop strain) in the critical point (the inner skin of the flank). This response can be decompsed into two parts:

- A cyclic elastic part:

$$
\epsilon_{\mathrm{cir}}=\alpha A(t)
$$

where $\epsilon_{\text {cir }}$ is the hoop strain.

- A cyclic plastic part given by the derivative with respect of time of the plastic ratchet $\dot{r}$ :

$$
\dot{r}= \begin{cases}0 & \text { if elastic evolution } \\ (\beta-\alpha) \dot{A}(t) & \text { if plastic evolution (closing) } \\ (\gamma-\alpha) \dot{A}(t) & \text { if plastic evolution (opening) }\end{cases}
$$

To obtain the global response, one must write the algorithm corresponding to the previous evolution and solve the problem numerically. Our method gives very good results with respect to the ones obtained by the FEM method. Furthermore, it is less time consuming.

\subsection{Evaluation of the plastic ratchet in the case of a seismic inputs}

Let us begin by evaluating of the plastic ratchet in the case of a centred cyclic loading opening/ closing as shown in (Fig. 8). Following our simplified model the evolution of the ratchet takes place when the amplitude of the imposed rotation is $>2 l$ as shown in Fig. 8. For every cycle, the plastic deformation (hoop strain) cycle is given as follows:

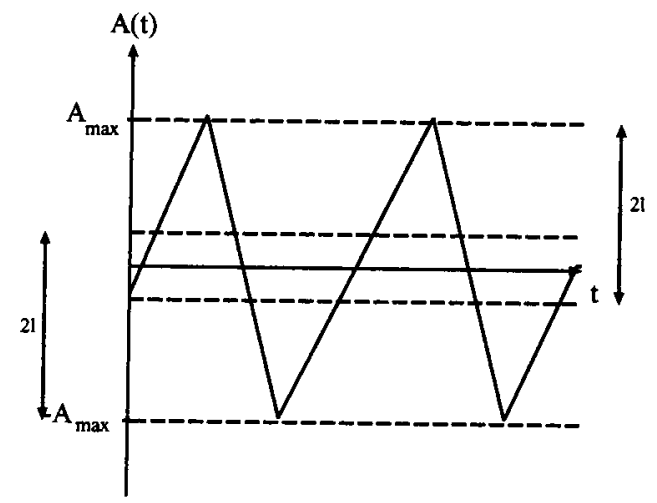

Fig. 8. Plastic evolution takes place for the bold parts of the curve. 
Table 2

Comparaison of the simplified method and FEM results

\begin{tabular}{lll}
\hline$m$ & Simplified ratchet $(\%)$ & FEM ratchet $(\%)$ \\
\hline 1 & 0 & 0 \\
2 & 3 & 2.9 \\
3 & 13.5 & 12.9 \\
4 & 24.5 & 21.7 \\
\hline
\end{tabular}

$\begin{cases}(\beta-\alpha)\left(-2 A^{\max }+2 l\right) & \text { for closing } \\ (\gamma-\alpha)\left(2 A^{\max }+2 l\right) & \text { for opening }\end{cases}$

Where $A^{\max }$ denotes the maximum of the absolute value of $A(t)$.

Considering Eq. (29) and taking into account the number of cycles $N$ yields the following expression of the final ratchet:

$r=2 N|\beta-\gamma|\left(A^{\max }-l\right)$

For the validation of our model, we calculated the ratchet due to a ten cycle loading with a maximum amplitude $A^{\max }=m-0.554 \quad(m=$ $\left(\max \mid a(t) / a_{\mathrm{y}}\right)$ using both the simplified formula and the FEM. In both cases we used the geometry and material parameters given in Table 1. The pressure was taken: $11.7 \mathrm{MPa}$. The results are given in Table 2. One can observe a good correlation between the two methods.

For a realistic seismic input like the one given in (Fig. 9), we can use the same procedure. In

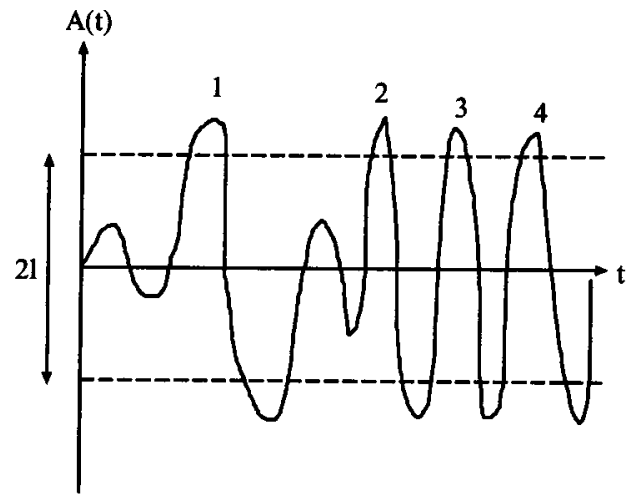

Fig. 9. Example of seismic input.
Table 3

Comparaison of the simplified method and FEM results for a seismic input

\begin{tabular}{llc}
\hline$m$ & Simplified ratchet $(\%)$ & FEM ratchet $(\%)$ \\
\hline 1.85 & 0.56 & 0.61 \\
2.59 & 3.8 & 3.73 \\
3 & 7.09 & 7.04 \\
3.5 & 12.28 & 12.23 \\
\hline
\end{tabular}

addition, approximating the loading history as Gaussien process leads the following formula:

$r=\sqrt{2 \pi} \sigma^{0} A^{\max }|\beta-\gamma|\left(1-\operatorname{erf}\left(\frac{1}{\sqrt{2} \sigma^{0} A^{\max }}\right)\right)$

where $\sigma^{0}$ is the standard deviation of the signal with normalised maximum amplitude, and erf is the error function defined by:

$\operatorname{erf}(z)=\frac{2}{\sqrt{\pi}} \int_{0}^{z} e^{-y^{2}} \mathrm{~d} y$

Table 3 summarises the results of a series of calculations of the ratchet by using either the simplified method (Eq. (31)) and the direct FEM. The predictions of the simplified method are very satisfactory.

\subsection{Extension of the simplified method for more realistic material behaviour}

Generally, steels present a non-linear plastic behaviour, which is not well described by the perfect plasticity. Non linear kinematic hardening effects are observed. They are often coupled with isotropic hardening. Such complex memory effects are observed in 316-L steel. To account for these effects, internal hardening parameters are necessary (Chaboche et al., 1979). The evolution of these parameters is very sensitive to the sequences of the applied loading so that the previous version of the simplified method cannot be applied directly. An extension is then necessary. Our main idea is based on the use of an equivalent-elastic perfectly plastic model in the sense that the final ratchet and fatigue damage should be the same as the ones predicted by the FEM calculation according to non linear hardening law. 


\subsubsection{Calculation of the equivalent elasticity limit}

Suppose that we have the resulting calculations of the FEM using a non-linear kinematic hardening law. The outcome of this calculation is the internal hoop strain in the inner flank of the pipe. We then look for an equivalent perfect plastic law, which gives, for the same level of loading, similar values of the final ratchet and fatigue damage as those given by the non-linear kinematic hardening behaviour. This equivalent law is subsequently characterised by a fictitious elastic limit $S_{\mathrm{y}}^{*}$. This limit is obtained by a parametric procedure based on a FEM calculations. The equivalent law must satisfy the following loading conditions:

$$
\left\{\begin{array}{c}
S_{\mathrm{y}}^{*}>S_{\mathrm{y}} \\
a_{\mathrm{y}}^{*}>a_{\mathrm{y}} \\
m^{*}<m \\
P^{*}<P
\end{array}\right.
$$

In Eq. (33) the symbol (*) denotes fictitious quantities. We recall that, $p$ represents the internal pressure, $p_{\mathrm{y}}$ the pressure level that gives the first plasticity without bending moment, $S_{\mathrm{y}}$ the elastic limit, $a_{\mathrm{y}}$ the angle corresponding to $S_{\mathrm{y}}, P$ and $m$ are adimensional constants that characterise the level of loading such that:

$$
\left\{\begin{array}{l}
p=P \times p_{\mathrm{y}} \\
a=m \times a_{\mathrm{y}}
\end{array}\right.
$$

Table 4

Results of the parametric calculation
The level of loading is determined in such a way that one obtains the same maximal opening amplitude of the elbow as with the FEM calculations. This parameter $m^{*}$ is then given by:

$m^{*}=\frac{m \times a_{\mathrm{y}}}{a_{\mathrm{y}}^{*}}$

\subsubsection{Results of the parametric analysis}

The elastic limit $S_{\mathrm{y}}^{*}$ is calculated using an iterative process: we look for the $S_{\mathrm{y}}^{*}$ that permits to obtain similar values of the ratchets given by the FEM and the simplified method by minimising their difference. Examples of results from the parametric calculations are presented in Table 4. Note that for the FEM calculations we submitted the pipe to a seismic input.

Using results from Table 4 one can determine a formal expression of the parameter $S_{\mathrm{y}}^{*}$ For example if $P=0.61$ we find that:

$S_{\mathrm{y}}^{*}=73.2 \times m+119.62$

\section{Fatigue analysis}

The last step in structural integrity assessment is the fatigue limit analysis. Let $N_{\text {fo }}$ denote the number of cycles to failure under cyclic strain only and $N_{\mathrm{f}}$ the number of cyclic to failure under the combination of cyclic strain and ratchet. The modified Masson-Coffin equation to take ac-

\begin{tabular}{|c|c|c|c|c|c|c|}
\hline$p(\mathrm{MPa})$ & $S_{\mathrm{y}}(\mathrm{MPa})$ & Level $m$ & level $P$ & FEM $(\%)$ & Equivalent (\%) & $S_{\mathrm{y}}^{*}$ \\
\hline 11.7 & 259 & 2 & 0.61 & 0.75 & 0.74 & 266 \\
\hline 11.7 & 259 & 2.3 & 0.61 & 0.97 & 0.975 & 288 \\
\hline 11.7 & 259 & 3 & 0.61 & 1.55 & 1.559 & 339 \\
\hline 11.7 & 259 & 3.63 & 0.61 & 2.1 & 2.08 & 386 \\
\hline 11.7 & 259 & 4 & 0.61 & 2.46 & 2.45 & 412 \\
\hline 11.7 & 230 & 3 & 0.68 & 1.26 & 1.27 & 318 \\
\hline 11.7 & 288 & 3 & 0.54 & 1.85 & 1.857 & 359 \\
\hline 15 & 230 & 3 & 0.87 & 1.90 & 1.881 & 344 \\
\hline 15 & 288 & 3 & 0.70 & 2.76 & 2.75 & 384 \\
\hline
\end{tabular}
count of ratcheting is then: 
Table 5

Comparaison of the simplified method and FEM results for a seismic input

\begin{tabular}{ll}
\hline Fatigue damage (FEM) & Fatigue damage (equivalent) \\
\hline $9.34 \mathrm{E}-3$ & $1.39 \mathrm{E}-2$ \\
$1.18 \mathrm{E}-2$ & $1.66 \mathrm{E}-2$ \\
$1.75 \mathrm{E}-2$ & $2.48 \mathrm{E}-2$ \\
$2.35 \mathrm{E}-2$ & $3.29 \mathrm{E}-2$ \\
$2.74 \mathrm{E}-2$ & $3.85 \mathrm{E}-2$ \\
$1.11 \mathrm{E}-2$ & $2.15 \mathrm{E}-2$ \\
$1.52 \mathrm{E}-2$ & $3.08 \mathrm{E}-2$ \\
$1.49 \mathrm{E}-2$ & $1.97 \mathrm{E}-2$ \\
$2.11 \mathrm{E}-2$ & $3.06 \mathrm{E}-2$ \\
$1.95 \mathrm{E}-2$ & $2.35 \mathrm{E}-2$ \\
$2.78 \mathrm{E}-2$ & $3.56 \mathrm{E}-2$
\end{tabular}

$\frac{N_{\mathrm{f}}}{N_{\mathrm{f}_{0}}}=\left(1-\frac{r_{\max }}{\epsilon_{\mathrm{f}}}\right)^{-1 / c}$

where $r_{\max }$ is the value of the ratchet given by the previous simplified calculation, $\epsilon_{\mathrm{f}}$ is the material ductility and $c$ is a material constant. The cumulative damage is given by

$D=\Sigma_{\text {cycles }} \frac{1}{N_{\mathrm{f}}}$

where the sum is performed on the number of cycles of the seismic input. Table 5 gives some predictions of the induced damage either by using non-linear kinematic hardening law and the simplified model. These results are satisfactory.

\section{Conclusion}

In this paper, a complete simplified method for fatigue-ratcheting analysis is proposed. It is applicable to materials with complex behaviour and does not necessitate lengthy FEM calculations. Our model is suitable to perform a great number of simulations necessary for a parametric sensitivity analysis that could be required to derive a design rule for a code of practice.

\section{Acknowledgements}

The authors would like to acknowledge Electricité De France (EDF) for their financial support. Thanks are due to V. Louche who carried out some Finite element calculations and to D.J. Boussa for fruitful discussions.

\section{References}

Boussa, D., DangYan, K., Labbé, P., Tang, M.T., 1996. Finite pure bending of curved pipes. Comput. Struct. 60, 10031012.

Boussa, D., et al. 1992. Flexion plane du tuyau coudé et endommagement sous séisme, Ph.D Thesis, ENPC Paris.

Boussa, D., DangYan, K., Labbé, P., Tang, M.T., 1994. Fatigue-Seismic Ratcheting Interactions in Pressurized Elbows. J. Press. Technol. 11G, 396-402.

Chaboche, J.L., Cordie, G., DangYan, K., 1979. Modelisation of the strain memory effect on the cycle hardening of 316-L stainless steel Proceedings. SMIRT 5, Vol. M.

Clarck, R.A., Reissner, E., 1951. Bending of curved tubes. Adv. Appl. Mech. 2, 93-122.

Coffin, L.F., 1970. The deformation and fracture of a ductile metal under superimposed cyclic and monotonic strain. In: ASTM STP 567. ASTM, Philadelphia, pp. 53-76.

Leconte, J., 1992. Chaudronnerie et tuyauteries industrielles, tome 1 Conception, Technique et Documentation. Lavoisier, Paris. 